\title{
Consensus of Fractional-Order Multiagent Systems with Double Integrator under Switching Topologies
}

\author{
Shiyun Shen, Wenjing Li, and Wei Zhu \\ Research Center of System Theory and Application, Chongqing University of Posts and Telecommunications, Chongqing 400065, China \\ Correspondence should be addressed to Wei Zhu; zhuwei@cqupt.edu.cn
}

Received 27 April 2017; Accepted 6 July 2017; Published 16 August 2017

Academic Editor: Antonio Iannizzotto

Copyright (C) 2017 Shiyun Shen et al. This is an open access article distributed under the Creative Commons Attribution License, which permits unrestricted use, distribution, and reproduction in any medium, provided the original work is properly cited.

\begin{abstract}
Due to the complexity of the practical environments, many systems can only be described with the fractional-order dynamics. In this paper, the consensus of fractional-order multiagent systems with double integrator under switching topologies is investigated. By applying Mittag-Leffler function, Laplace transform, and dwell time technique, a sufficient condition on consensus is obtained. Finally, a numerical simulation is presented to illustrate the effectiveness of the theoretical result.
\end{abstract}

\section{Introduction}

In recent years, cooperative control of multiagent systems has received compelling attention due to its wide applications such as minisatellites [1], unmanned vehicle [2], deployment [3], and rendezvous [4]. A significant and fundamental problem for cooperative control of multiagent systems (MASs) is consensus, which has broad applications in so many fields such as multivehicle systems [5], swarms, and flocks [6]. It was found that many MASs can be explained in the framework of fractional-order dynamics and the distributed control of fractional-order multiagent systems is becoming an important issue.

Consensus of FMASs was early discussed by Cao and Ren in [7-9] and then attracted many researchers' attention. In [10], by using the matrix theory, graph theory and the frequency-domain analysis method, the control algorithm with absolute damping and communication delay was proposed to discuss the distributed formation control of FMASs. In [11], a distributed control law with a constant reference state was given, which ensured the consensus of FMASs with a time-varying reference state. In [12], the fractional Lyapunov direct method and Mittag-Leffler stability were used to study the consensus of nonlinear FMASs with directed topology, some sufficient conditions were given to guarantee the consensus of nonlinear FMASs with/without a virtual leader. In [13], based on the connectivity of the graph and Riccati equation, the control gain matrix was designed and a sufficient condition on leader-following consensus of FMASs with general linear models was obtained. In [14, 15], the authors studied the leader-following consensus of FMASs with nonlinear dynamics by Lyapunov direct method, respectively. In [16], based on the properties of Mittag-Leffler function, matrix theory, and stability theory of fractionalorder differential equations, some sufficient conditions on consensus were derived to guarantee the consensuses of linear and nonlinear FMASs for any bounded input time delay. In [17], a distributed observer-type protocol was proposed to study the consensus of FMASs by applying the properties of the Kronecker product, Mittag-Leffler function, and Laplace transform.

Most of the existing literatures about FMASs consensus focused on the fixed topology. In fact, when the multiagent system moves harmoniously, communications between agents are usually intermittent due to external disturbances, limited communication range, or the instability of wireless communication itself. The consensus of FMASs under switching topologies is a more important and challenging problem. In this paper, the consensus of FMASs with double integrator under switching topologies is investigated. By using Mittag-Leffler function, Laplace transform, and dwell time technique, a sufficient condition on consensus is presented to guarantee the consensus of the system. 


\section{Preliminaries}

2.1. Graph Theory. To describe the switching topologies, we define a piecewise constant switching signal $\sigma(t):[0, \infty) \rightarrow$ $\Lambda=\{1,2, \ldots, N\}$ represented by $\{p\}$ according to $\left[t_{q-1}, t_{q}\right) \rightarrow$ $p \in \Lambda$, where $q=1,2, \ldots$ and $N$ is the total number of all possible interconnection graphs.

The switching topologies of information exchange between $n$ agents are described by $\mathscr{G}_{p}=\left(\mathscr{V}, \mathscr{E}_{p}, A_{p}\right), p \in \Lambda$, where $\mathscr{V}=\{1,2, \ldots, n\}$ and $\mathscr{E}_{p} \subset \mathscr{V} \times \mathscr{V}$ are the sets of vertices and edges of the graph $\mathscr{G}_{p}$, respectively. The adjacency matrices $A_{p}=\left(a_{i j}^{p}\right)_{n \times n}$, where $a_{i j}^{p}$ is the weight of the directed edge $(j, i) \in \mathscr{E}_{p}$ in the graph $\mathscr{G}_{p}$ and $j$ and $i$ are called the initial and terminal nodes, respectively, which means that node $i$ can receive information from node $j$. $a_{i j}^{p}>0$ if and only if there is a directed edge $(j, i)$ in $\mathscr{G}_{p}$. Moreover, we assume $a_{i i}^{p}=0$ for all $i=1,2, \ldots, n, p \in \Lambda$. The degree matrices $D_{p}=\operatorname{diag}\left\{d_{1}^{p}, \ldots, d_{n}^{p}\right\}$ are diagonal matrices, whose diagonal elements are given by $d_{i}^{p}=\sum_{j=1}^{n} a_{i j}^{p}$. The Laplacian matrix of the weighted digraph is defined as $L_{p}=D_{p}-A_{p}$ for every $p$. The set of neighbors of node $i$ is denoted by $\mathscr{N}_{i}^{p}=\left\{j \in \mathscr{V}:(j, i) \in \mathscr{E}_{p}\right\}$.

2.2. Caputo Fractional Derivative. The Caputo fractional derivative is defined as follows:

$$
{ }_{a}^{C} D_{t}^{\alpha} f(t)=\frac{1}{\Gamma(n-\alpha)} \int_{a}^{t} \frac{f^{(n)}(\tau)}{(t-\tau)^{\alpha-n+1}} d \tau,
$$

$$
n-1<\alpha \leq n
$$

where $\alpha>0$ is the fractional order, $n$ is any given natural number, and $\Gamma(\cdot)$ is the Gamma function defined as $\Gamma(\tau)=$ $\int_{0}^{\infty} t^{\tau-1} e^{-t} d t$. A simple notion $D^{\alpha} f(t)$ will be used to denote ${ }_{a}^{C} D_{t}^{\alpha} f(t)$ if no confusion has arisen.

Let $\mathscr{L}\{\cdot\}$ denote the Laplace transform of a function. Then we have

$$
\mathscr{L}\left\{D^{\alpha} f(t)\right\}=s^{\alpha} F(s)-s^{\alpha-1} f\left(t_{0}\right), \quad \alpha \in(0,1],
$$

where $F(s)$ is the Laplace transform of $f(t)$.

Similar to the exponential function frequently used in the solutions of integer-order systems, a function frequently used in the solutions of fractional-order systems is the MittagLeffler function.

Definition 1 (see [18]). The two-parameter function of the Mittag-Leffler type is defined by

$$
E_{\alpha, \beta}(z)=\sum_{k=0}^{\infty} \frac{z^{k}}{\Gamma(k \alpha+\beta)},
$$

where $\alpha>0, \beta>0$, and $z$ is a complex number.

In particular, let $\beta=1$ and $\alpha>0$; (3) becomes

$$
E_{\alpha, 1}(z) \triangleq E_{\alpha}(z)=\sum_{k=0}^{\infty} \frac{z^{k}}{\Gamma(k \alpha+1)} .
$$

2.3. System Model Description. Consider the following fractional-order double-integrator multiagent systems with $n$ agents:

$$
\begin{aligned}
& D^{\alpha} x_{i}(t)=v_{i}(t), \\
& D^{\alpha} v_{i}(t)=u_{i}(t),
\end{aligned}
$$

$$
t \geq t_{0}, i \in \Delta \text {, }
$$

where $0<\alpha \leq 1, \Delta=\{1,2, \ldots, n\}, x_{i}(t), v_{i}(t) \in R$, and $u_{i}(t) \in$ $R$ are the states and control input of the $i$ th agent, respectively. Assume that the initial states of each agent $i$ are denoted by $x_{i}\left(t_{0}\right)$ and $v_{i}\left(t_{0}\right)$.

Definition 2. For multiagent systems (5), the protocols $u_{i}(t)$ are said to solve the consensus if and only if, for any initial values, the states of the agents satisfy

$$
\begin{aligned}
\lim _{t \rightarrow \infty}\left\|x_{j}(t)-x_{i}(t)\right\| & =0, \quad i, j \in \Delta, \\
\lim _{t \rightarrow \infty}\left\|D^{\alpha} x_{j}(t)-D^{\alpha} x_{i}(t)\right\| & =0, \quad i, j \in \Delta .
\end{aligned}
$$

To study the consensus of multiagent systems (5), the following consensus control protocol will be used:

$$
u_{i}(t)=-\sum_{j \in \mathcal{N}_{i}^{p}, j \neq i} a_{i j}^{p}\left(x_{i}(t)-x_{j}(t)\right)-k D^{\alpha} x_{i}(t),
$$

$i \in \Delta$,

where $k>0$ is the control parameter to be designed later.

Let $x(t)=\left(x_{1}(t), x_{2}(t), \ldots, x_{n}(t)\right)^{T}, v(t)=\left(v_{1}(t), v_{2}(t)\right.$, $\left.\ldots, v_{n}(t)\right)^{T}$. Then (5) can be rewritten in the following compact form:

$$
\begin{aligned}
& D^{\alpha} x(t)=v(t) \\
& D^{\alpha} v(t)=-L_{p} x(t)-k v(t) .
\end{aligned}
$$

Furthermore, let $\mathbf{1}_{n-1}^{T}=\underbrace{[1, \ldots, 1]}_{n-1}, \widetilde{x}(t)=S x(t), \widetilde{v}(t)=S v(t)$, where

$$
S=\left(\begin{array}{cc}
1 & 0 \\
-\mathbf{1}_{\mathbf{n}-\mathbf{1}} & I_{n-1}
\end{array}\right)
$$

that is, $\widetilde{x}(t)=\left(x_{1}(t), x_{2}(t)-x_{1}(t), \ldots, x_{n}(t)-x_{1}(t)\right)^{T}, \widetilde{v}(t)=$ $\left(v_{1}(t), v_{2}(t)-v_{1}(t), \ldots, v_{n}(t)-v_{1}(t)\right)^{T}$. Then, by (8), we have

$$
\begin{aligned}
& D^{\alpha} \widetilde{x}(t)=\widetilde{v}(t), \\
& D^{\alpha} \widetilde{v}(t)=-\left(S L_{p} S^{-1}\right) \widetilde{x}(t)-k \widetilde{v}(t) .
\end{aligned}
$$

Define $\widehat{x}(t)=\left(x_{2}(t)-x_{1}(t), \ldots, x_{n}(t)-x_{1}(t)\right)^{T}, \widehat{v}(t)=\left(v_{2}(t)-\right.$ $\left.v_{1}(t), \ldots, v_{n}(t)-v_{1}(t)\right)^{T}$. Then, (10) can be decoupled as follows:

$$
\begin{aligned}
& D^{\alpha} \widehat{x}(t)=\widehat{v}(t), \\
& D^{\alpha} \widehat{v}(t)=-\widetilde{L}_{p} \widehat{x}(t)-k \widehat{v}(t),
\end{aligned}
$$


where

$$
\widetilde{L}_{p}=\left(\begin{array}{cccc}
d_{2}^{p}+a_{12}^{p} & a_{13}^{p}-a_{23}^{p} & \cdots & a_{1 n}^{p}-a_{2 n}^{p} \\
a_{12}^{p}-a_{32}^{p} & d_{3}^{p}+a_{13}^{p} & \cdots & a_{1 n}^{p}-a_{3 n}^{p} \\
\vdots & \vdots & \ddots & \vdots \\
a_{12}^{p}-a_{n 2}^{p} & a_{13}^{p}-a_{n 3}^{p} & \cdots & d_{n}^{p}+a_{1 n}^{p}
\end{array}\right) .
$$

Let $\varepsilon(t)=\left(\hat{x}^{T}(t), \widehat{v}^{T}(t)\right)^{T}$; (11) can be rewritten in the following compact form:

$$
D^{\alpha} \varepsilon(t)=\mathscr{C}_{p} \varepsilon(t)
$$

where

$$
\mathscr{C}_{p}=\left(\begin{array}{cc}
0 & I_{n-1} \\
-\widetilde{L}_{p} & -k I_{n-1}
\end{array}\right) .
$$

\section{Main Results}

Let $Q_{s}$ be the set over which there is a directed spanning tree for any $\mathscr{G}_{p} \in \mathbb{Q}_{s}$ and $\mathbb{Q}_{u}$ be the set over which there is no directed spanning tree for any $\mathscr{G}_{p} \in \mathbb{Q}_{u}$. Denote the cardinality of $\Lambda$ by $|\Lambda|$. Without loss of generality, we assume that $\mathbb{Q}_{s}=\left\{\mathscr{G}_{p}, p=1,2, \ldots, v\right\}$ and $\mathbb{Q}_{u}=\left\{\mathscr{G}_{p}, p=v+\right.$ $1, v+2, \ldots,|\Lambda|\}$ for some $v \in \Lambda$. Assume that the activating topology on $\left[t_{q-1}, t_{q}\right), q=1,2, \ldots$, is $\mathscr{G}_{p}$, where $p \in \Lambda$ and $p=q \bmod |\Lambda|$, and set $p=|\Lambda|$ if $p=0$. Let $t_{q}-t_{q-1} \geq T^{*}$, where $T^{*}$ is called to be the dwell time of the switching signal $\sigma(t)$. During each of intervals, the interconnection topology does not change. For any $t>t_{0}$, let $T_{p}\left(t_{0}, t\right)$ denote the total activation time of $\mathscr{G}_{p}, p \in \Lambda$ and $T_{s}(t)=\sum_{p=1}^{v} T_{p}\left(t_{0}, t\right)$, $T_{u}(t)=\sum_{p=v+1}^{|\Lambda|} T_{p}\left(t_{0}, t\right)$.

Based on Lemma 3.1 in [17], with a mild revision, we have the following.

Lemma 3. All the eigenvalues of matrix $\widetilde{L}_{p}$ have positive real parts if and only if $\mathscr{G}_{p} \in \mathbb{Q}_{s}$.

Lemma 4 (see [19]). Let

$$
Q=\left(\begin{array}{cc}
0 & I \\
-H & -k I
\end{array}\right)
$$

Assume that the control parameter $k$ satisfies $k^{2}>$ $\left(\max _{\mu \in \rho(H)}\{|\operatorname{Im} \mu|\}\right)^{2} / \min _{\mu \in \rho(H)} \operatorname{Re} \mu,\left(\min _{\mu \in \rho(H)} \operatorname{Re} \mu \neq 0\right)$, where $\rho(H)$ denotes the set of all eigenvalues of $H$. Then $\max _{\theta \in \rho(Q)} \operatorname{Re} \theta<0$ if and only if all eigenvalues of $H$ have positive real parts.

By Lemma 2.2 in [20], with a mild revision, we have the following.

Lemma 5. There exist finite real constants $h \geq 1$ such that, for any $0<\alpha<1$ and $\beta>0$,

$$
\left\|E_{\alpha, \beta}\left(A t^{\alpha}\right)\right\| \leq h\left\|e^{A t}\right\| .
$$

In addition, if $A$ is a stable matrix, then there are some real constants $K \geq 1, \lambda>0$ such that

$$
\left\|E_{\alpha, \beta}\left(A t^{\alpha}\right)\right\| \leq K e^{-\lambda t}
$$

Theorem 6. Consider the fractional-order double-integrator multiagent systems (5) under the control protocol (7) and take $k^{2}>\max _{1 \leq p \leq v}\left\{\left(\max _{\mu \in \rho\left(\widetilde{L}_{p}\right)}\{|\operatorname{Im} \mu|\}\right)^{2} / \min _{\mu \in \rho\left(\widetilde{L}_{p}\right)} \operatorname{Re} \mu\right\}$. Then, the consensus can be achieved if the switching signal satisfying the dwell time condition (28) and the following inequality holds:

$$
\inf _{t>t_{0}} \frac{T_{s}(t)}{T_{u}(t)}>\frac{\gamma}{\eta},
$$

where $\eta=\min _{1 \leq p \leq v}\left\{\eta_{p}\right\}>0$ and $\gamma$ satisfies $\max _{v+1 \leq p \leq|\Lambda|}\left\{\left\|\mathscr{C}_{p}\right\|\right\}<\gamma<\infty$ and $\gamma\left(\gamma-\left\|\mathscr{C}_{p}\right\|\right)>0, p=$ $v+1, v+2, \ldots,|\Lambda|$.

Proof. For $t \in\left(t_{p-1}, t_{p}\right]$, taking the Laplace transform on (13) and using the Laplace transform (2) with $\alpha \in(0,1]$, we have

$$
\mathscr{L}\{\varepsilon(t)\}=\frac{s^{\alpha-1} \varepsilon\left(t_{p-1}\right)}{I s^{\alpha}-\mathscr{C}_{p}} .
$$

Then, taking the inverse Laplace transform of (19), one can obtain that

$$
\varepsilon(t)=E_{\alpha}\left(\mathscr{C}_{p}\left(t-t_{p-1}\right)^{\alpha}\right) \varepsilon\left(t_{p-1}\right) .
$$

For $p=1,2, \ldots, v$, since the communication graph $\mathscr{G}_{p}$ has a directed spanning tree, by Lemma 3, all the eigenvalues of matrix $\widetilde{L}_{p}$ have positive real parts. It follows from the choice of parameter $k$ and Lemma 4 that $\max _{\theta \in \rho\left(\mathscr{C}_{p}\right)} \operatorname{Re} \theta<0$. Hence, $\mathscr{C}_{p}$ is a stable matrix. It follows from Lemma 5 that there exist constants $K_{p} \geq 1$ and $\eta_{p}>0$ such that

$$
\begin{aligned}
\|\varepsilon(t)\| & =\left\|E_{\alpha}\left(\mathscr{C}_{p}\left(t-t_{p-1}\right)^{\alpha}\right) \varepsilon\left(t_{p-1}\right)\right\| \\
& \leq K_{p} e^{-\eta_{p}\left(t-t_{p-1}\right)}\left\|\varepsilon\left(t_{p-1}\right)\right\|, \quad t \in\left(t_{p-1}, t_{p}\right] .
\end{aligned}
$$

For $p=v+1, v+2, \ldots,|\Lambda|$, since $\max _{v+1 \leq p \leq|\Lambda|}\left\{\left\|\mathscr{C}_{p}\right\|\right\}<\gamma<\infty$ and $\gamma\left(\gamma-\left\|\mathscr{C}_{p}\right\|\right)>0$, obviously, $\gamma$ is well defined. It follows from Lemma 5 that there exist constants $h_{p}>0$ such that

$$
\begin{aligned}
\|\varepsilon(t)\| & =\left\|E_{\alpha}\left(\mathscr{C}_{p}\left(t-t_{p-1}\right)^{\alpha}\right) \varepsilon\left(t_{p-1}\right)\right\| \\
& \leq h_{p}\left\|e^{\mathscr{C}_{p}\left(t-t_{p-1}\right)}\right\|\left\|\varepsilon\left(t_{p-1}\right)\right\| \\
& \leq h_{p} e^{\left\|\mathscr{C}_{p}\right\|\left(t-t_{p-1}\right)}\left\|\mathcal{E}\left(t_{p-1}\right)\right\| \\
& \leq h_{p} e^{\gamma\left(t-t_{p-1}\right)}\left\|\varepsilon\left(t_{p-1}\right)\right\|, \quad t \in\left(t_{p-1}, t_{p}\right] .
\end{aligned}
$$

Then, for $t \in\left(t_{v-1}, t_{v}\right]$, by (21), we obtain that

$$
\begin{aligned}
& \|\mathcal{E}(t)\| \leq K_{v} e^{-\eta_{v}\left(t-t_{v-1}\right)}\left\|\mathcal{E}\left(t_{v-1}\right)\right\| \leq K_{1} K_{2} \\
& \cdots K_{v} e^{-\eta_{1}\left(t_{1}-t_{0}\right)} \cdots e^{-\eta_{v-1}\left(t_{v-1}-t_{v-2}\right)} e^{-\eta_{v}\left(t-t_{v-1}\right)}\left\|\mathcal{E}\left(t_{0}\right)\right\| \\
& =K_{1} K_{2} \cdots K_{v} e^{-\sum_{p=1}^{v-1} \eta_{p}\left(t_{p}-t_{p-1}\right)-\eta_{v}\left(t-t_{v-1}\right)}\left\|\mathcal{E}\left(t_{0}\right)\right\| .
\end{aligned}
$$


For $t \in\left(t_{v}, t_{v+1}\right]$, by (22), one can derive that

$$
\begin{aligned}
& \|\mathcal{E}(t)\| \leq h_{v+1} e^{\gamma\left(t-t_{v}\right)}\left\|\mathcal{E}\left(t_{v}\right)\right\| \\
& \quad \leq K_{1} K_{2} \cdots K_{v} h_{v+1} e^{-\sum_{p=1}^{v} \eta_{p}\left(t_{p}-t_{p-1}\right)} e^{\gamma\left(t-t_{v}\right)}\left\|\mathcal{E}\left(t_{0}\right)\right\|,
\end{aligned}
$$

and, then, for $t \in\left(t_{|\Lambda|-1}, t_{|\Lambda|}\right]$, we obtain that

$$
\begin{aligned}
& \|\mathcal{E}(t)\| \leq h_{|\Lambda|} e^{\gamma\left(t-t_{|\Lambda|-1}\right)}\left\|\mathcal{E}\left(t_{|\Lambda|-1}\right)\right\| \leq K_{1} K_{2} \\
& \cdots K_{v} h_{v+1} h_{v+2} \\
& \cdots h_{|\Lambda|} e^{-\sum_{p=1}^{v} \eta_{p}\left(t_{p}-t_{p-1}\right)} e^{\sum_{p=v+1}^{|\Lambda|-1} \gamma\left(t_{p}-t_{p-1}\right)+\gamma\left(t-t_{|\Lambda|-1}\right)}\left\|\mathcal{E}\left(t_{0}\right)\right\| \\
& \leq K_{1} K_{2} \cdots K_{v} h_{v+1} h_{v+2} \\
& \cdots h_{|\Lambda|} e^{-\sum_{p=1}^{v} \eta_{p}\left(t_{p}-t_{p-1}\right)} e^{\sum_{p=v+1}^{|\Lambda|} \gamma\left(t_{p}-t_{p-1}\right)}\left\|\mathcal{E}\left(t_{0}\right)\right\| .
\end{aligned}
$$

In general, noticing that $\mathscr{G}_{p}$ is finite, using the definitions of $T_{p}\left(t_{0}, t\right), T_{s}(t), T_{u}(t)$, and $(25)$, it is clear that

$$
\begin{aligned}
\|\mathcal{E}(t)\| & \leq \omega^{\left(t-t_{0}\right) / T^{*}} e^{-\sum_{p=1}^{v} \eta_{p} T_{p}\left(t_{0}, t\right)} e^{\sum_{p=v+1}^{|\Lambda|} \gamma T_{p}\left(t_{0}, t\right)}\left\|\mathcal{E}\left(t_{0}\right)\right\| \\
& \leq e^{\left(\left(t-t_{0}\right) / T^{*}\right) \ln \omega}\left\|\mathcal{E}\left(t_{0}\right)\right\| e^{-\eta T_{s}(t)} e^{\gamma T_{u}(t)} \\
& \leq e^{\left(\ln \omega / T^{*}\right)\left(t-t_{0}\right)}\left\|\mathcal{E}\left(t_{0}\right)\right\| e^{-\eta T_{s}(t)+\gamma T_{u}(t)}, \quad t>t_{0},
\end{aligned}
$$

where $\omega=\max _{p \in \Lambda}\left\{K_{p}, h_{p}\right\}$.

According to $\inf _{t>t_{0}}\left(T_{s}(t) / T_{u}(t)\right)>\gamma / \eta$, there exists a constant $\sigma>0$ such that $(\gamma+\sigma) T_{u}(t) \leq(\eta-\sigma) T_{s}(t)$, so $-\eta T_{s}(t)+\gamma T_{u}(t) \leq-\sigma\left(T_{u}(t)+T_{s}(t)\right)=-\sigma\left(t-t_{0}\right)$. By $(26)$, we have

$$
\|\mathcal{E}(t)\| \leq\left\|\mathcal{E}\left(t_{0}\right)\right\| e^{\left(\ln \omega / T^{*}-\sigma\right)\left(t-t_{0}\right)}, \quad t>t_{0} .
$$

Thus, for the switching signal satisfying the dwell time condition,

$$
\frac{\ln \omega}{\sigma}<T^{*} \leq t_{q}-t_{q-1}, \quad q=1,2, \ldots
$$

we can derive that $\ln \omega / T^{*}-\sigma<0$, which implies that the consensus can be achieved asymptotically.

\section{A Numerical Example and Simulation}

Consider the fractional-order multiagent systems with $\alpha=$ 0.8. The switching topologies are shown in Figure 1.
For simplicity, we assume $a_{i j}^{p}=1$ if $(j, i)$ in $\mathscr{G}_{p}, p=$ $1,2,3,4$. Then, by the definition of $\widetilde{L}_{p}$, we have

$$
\widetilde{L}_{1}=\left(\begin{array}{cccc}
1 & 0 & 0 & 1 \\
0 & 2 & -1 & 0 \\
0 & -1 & 2 & 1 \\
0 & -1 & 0 & 2
\end{array}\right) \text {, }
$$$$
\widetilde{L}_{2}=\left(\begin{array}{cccc}
1 & 0 & 0 & 0 \\
0 & 1 & 0 & -1 \\
0 & 0 & 0 & 0 \\
0 & 0 & -1 & 2
\end{array}\right) \text {, }
$$$$
\widetilde{L}_{3}=\left(\begin{array}{cccc}
1 & 0 & 0 & 1 \\
1 & 2 & -1 & 0 \\
1 & 0 & 0 & 1 \\
1 & -1 & 0 & 2
\end{array}\right) \text {, }
$$

$$
\widetilde{L}_{4}=\left(\begin{array}{cccc}
1 & 0 & 0 & 0 \\
1 & 1 & 0 & -1 \\
1 & 0 & 1 & 0 \\
1 & -1 & -1 & 2
\end{array}\right) .
$$

By simple computation, we have $\rho\left(\widetilde{L}_{1}\right)=1,3.3247,1.3376+$ $0.5623 \sqrt{-1}, 1.3376-0.5623 \sqrt{-1}, \rho\left(\widetilde{L}_{4}\right)=2.6180,0.3820,1$, 1 ; then $k^{2}>0.828$. It is clear that there is a directed spanning tree in $\mathscr{G}_{1}$ and $\mathscr{G}_{4}$. Then, the consensus can be achieved for some $T^{*}$ and switching signal $\sigma(t)$ satisfying (18).

Let $T^{*}=10, k=3$, and the initial states be $x_{1}(0)=8$, $x_{2}(0)=-10, x_{3}(0)=-5, x_{4}(0)=16, x_{5}(0)=-7, v_{1}(0)=-5$, $v_{2}(0)=1, v_{3}(0)=-9, v_{4}(0)=4$, and $v_{5}(0)=15$. The control $u_{i}(t)$ and switching sequence are depicted in Figure 2. The state responses $x_{i}(t)$ and $v_{i}(t)$ are depicted in Figure 3, which shows the consensus is achieved asymptotically.

Remark 7. From the above simulation, it is found that the condition (18) is conservative. But within this frame of this paper, the condition (18) can hardly be improved. Sharpening the condition may require a new method. We leave this for further investigation.

\section{Conclusions}

The consensus problem of fractional-order multiagent systems with double integrator under switching topologies is investigated, where the fractional order satisfies $0<\alpha \leq 1$. Consensus algorithms with a control parameter are designed. Based on the Mittag-Leffler function, Laplace transform, and dwell time technique, a sufficient condition is obtained, which ensures that the consensus of fractional-order multiagent systems can achieved asymptotically. The consensus problem of fractional-order multiagent systems with jointly connected topologies will be discussed in future study. 


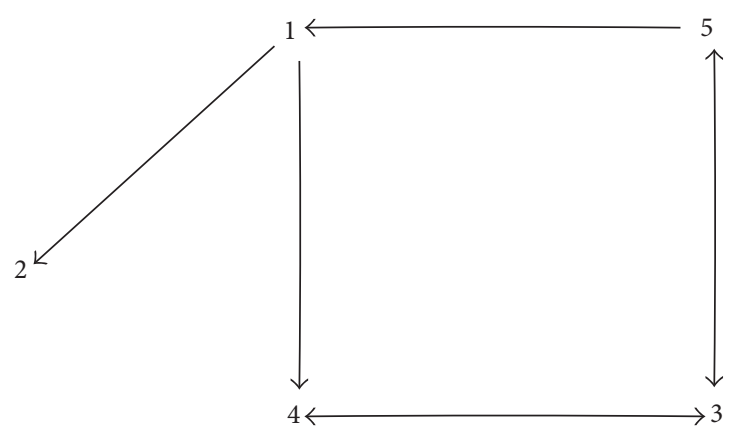

$\mathscr{G}_{1}$

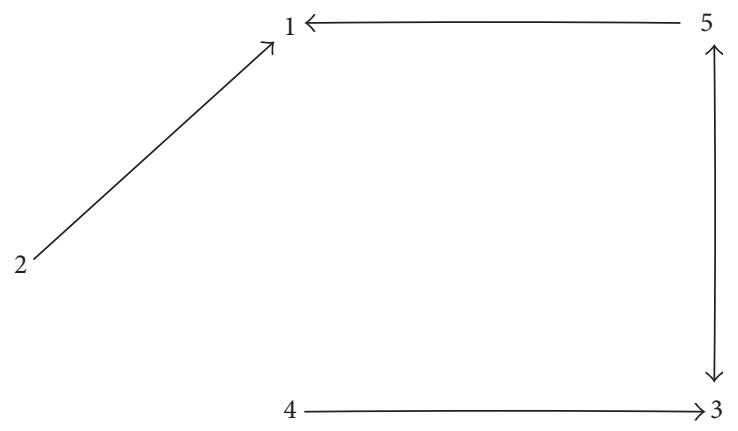

$\mathscr{G}_{3}$

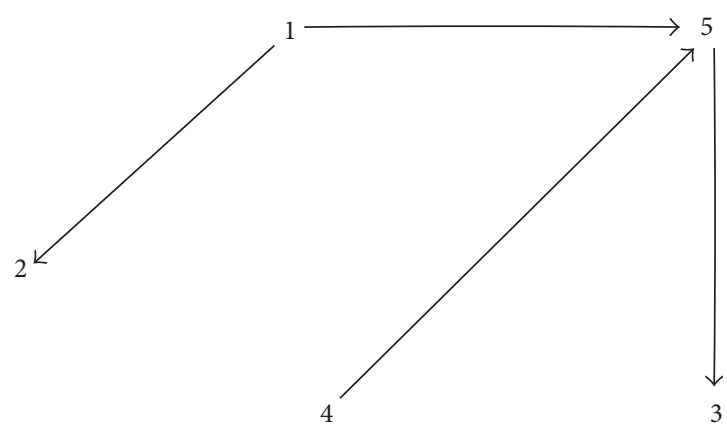

$\mathscr{G}_{2}$

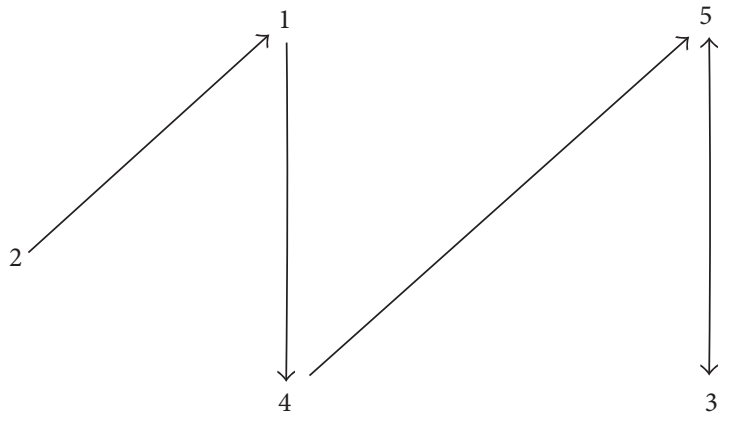

$\mathscr{G}_{4}$

Figure 1: Switching topologies: $\mathscr{G}_{i}, i=1,2,3,4$.
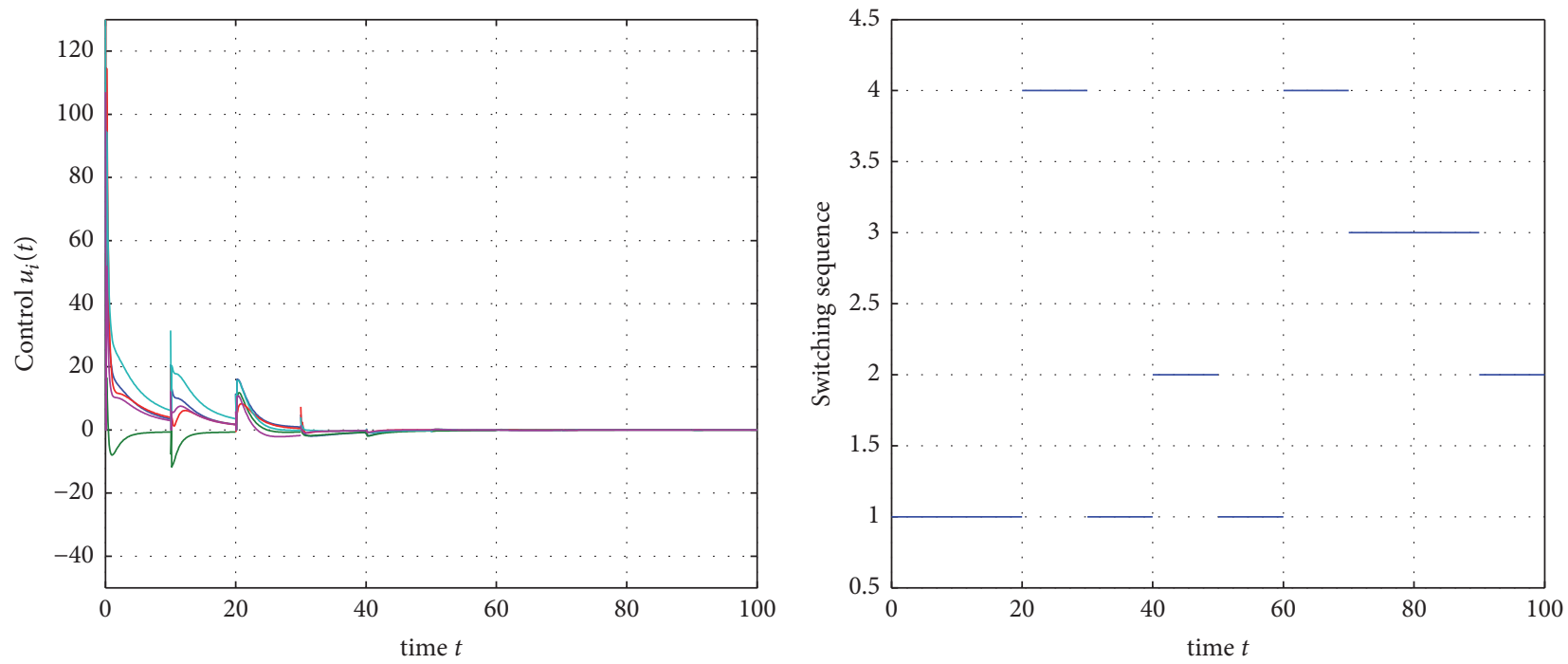

FIGURE 2: Control $u_{i}(t)$ and switching sequence.

\section{Conflicts of Interest}

The authors declare that there are no conflicts of interest regarding the publication of this paper and the mentioned received funding does not lead to any conflicts of interest regarding the publication.

\section{Acknowledgments}

This work is supported partly by National Natural Science Foundation of China under Grants 61673080 and 61403314, partly by Training Programme Foundation for the Talents of Higher Education by Chongqing Education Commission, 

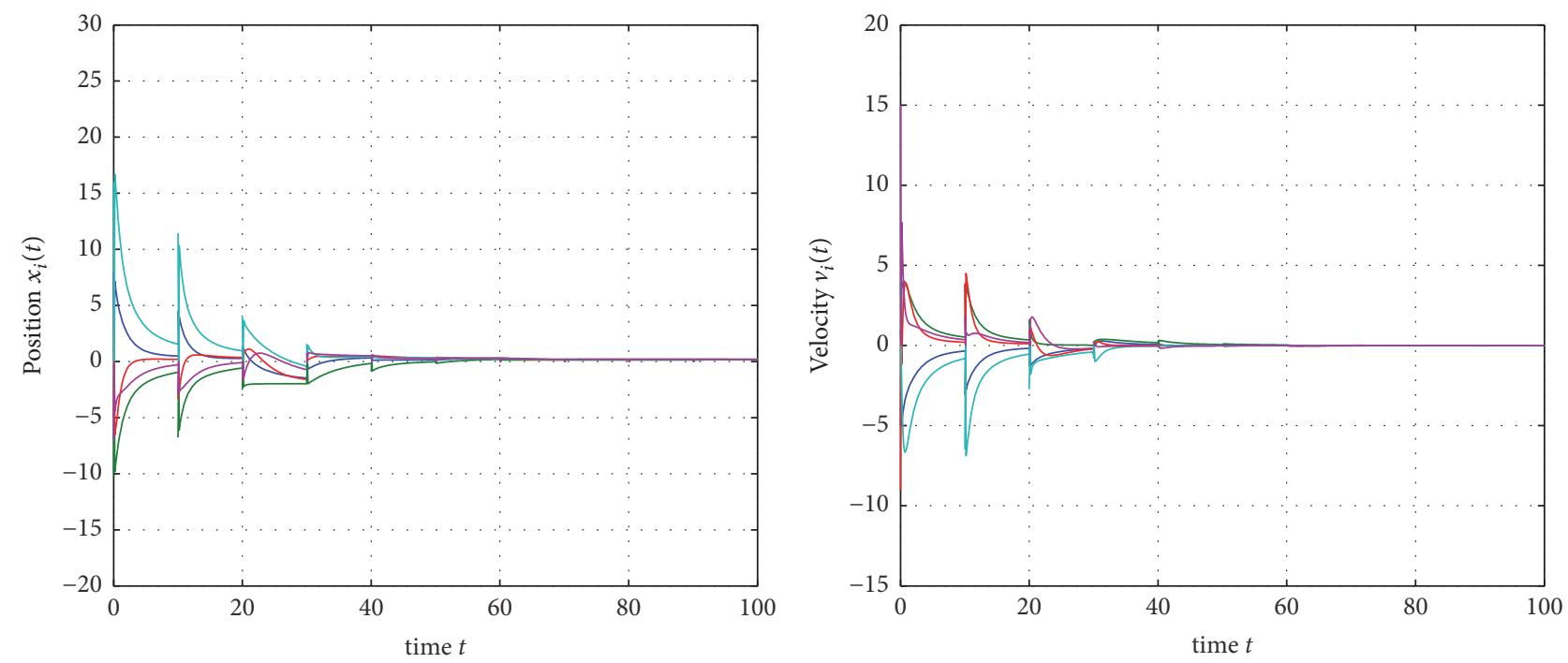

FIGURE 3: State responses $x_{i}(t)$ and $v_{i}(t)$ of each agent.

and partly by Innovation Team Project of Chongqing Education Committee under Grant CXTDX201601019.

\section{References}

[1] Y. Sun, "Average consensus in networks of dynamic agents with uncertain topologies and time-varying delays," Journal of the Franklin Institute. Engineering and Applied Mathematics, vol. 349, no. 3, pp. 1061-1073, 2012.

[2] W. Ren and E. Atkins, "Distributed multi-vehicle coordinated control via local information exchange," International Journal of Robust and Nonlinear Control, vol. 17, no. 10-11, pp. 10021033, 2007.

[3] C. Nowzari and J. Cortés, "Self-triggered coordination of robotic networks for optimal deployment," Automatica. A Journal of IFAC, the International Federation of Automatic Control, vol. 48, no. 6, pp. 1077-1087, 2012.

[4] Y. Fan, G. Feng, Y. Wang, and C. Song, "Distributed eventtriggered control of multi-agent systems with combinational measurements," Automatica. A Journal of IFAC, the International Federation of Automatic Control, vol. 49, no. 2, pp. 671675, 2013.

[5] J. A. Fax and R. M. Murray, "Information flow and cooperative control of vehicle formations," Institute of Electrical and Electronics Engineers. Transactions on Automatic Control, vol. 49, no. 9, pp. 1465-1476, 2004.

[6] H. G. Tanner, A. Jadbabaie, and G. J. Pappas, "Flocking in fixed and switching networks," Institute of Electrical and Electronics Engineers. Transactions on Automatic Control, vol. 52, no. 5, pp. 863-868, 2007.

[7] Y. Cao and W. Ren, "Distributed formation control for fractional-order systems: dynamic interaction and absolute/relative damping," Systems and Control Letters, vol. 59, no. 3-4, pp. 233-240, 2010.

[8] Y. C. Cao, Y. Li, W. Ren, and Y. Q. Chen, "Distributed coordination of networked fractional-order systems," IEEE Transactions on Systems, Man, and Cybernetics B: Cybernetics, vol. 40, no. 2, pp. 362-370, 2010.
[9] Y. Cao, Y. Li, W. Ren, and Y. Q. Chen, "Distributed coordination algorithms for multiple fractional-order systems," in Proceedings of the 47th IEEE Conference on Decision and Control (CDC '08), pp. 2920-2925, Cancun, Mexico, December 2008.

[10] J. Bai, G. Wen, A. Rahmani, and Y. Yu, "Distributed formation control of fractional-order multi-agent systems with absolute damping and communication delay," International Journal of Systems Science. Principles and Applications of Systems and Integration, vol. 46, no. 13, pp. 2380-2392, 2015.

[11] J. Bai, G. Wen, A. Rahmani, X. Chu, and Y. Yu, "Consensus with a reference state for fractional-order multi-agent systems," International Journal of Systems Science. Principles and Applications of Systems and Integration, vol. 47, no. 1, pp. 222-234, 2016.

[12] P. Gong, "Distributed consensus of non-linear fractional-order multi-agent systems with directed topologies," IET Control Theory \& Applications, vol. 10, no. 18, pp. 2515-2525, 2016.

[13] W. Zhu, M. Wang, and C. Yang, "Leader-following consensus of fractional-order multi-agent systems with general linear models," in Proceedings of the 2014 11th World Congress on Intelligent Control and Automation, WCICA 2014, pp. 3493-3496, IEEE, Shenyang, China, July 2014.

[14] G. Ren, Y. Yu, and S. Zhang, "Leader-following consensus of fractional nonlinear multiagent systems," Mathematical Problems in Engineering, Article ID 919757, Art. ID 919757, 8 pages, 2015.

[15] Z. Yu, H. Jiang, and C. Hu, "Leader-following consensus of fractional-order multi-agent systems under fixed topology," Neurocomputing, vol. 149, pp. 613-620, 2015.

[16] W. Zhu, B. Chen, and J. Yang, "Consensus of fractional-order multi-agent systems with input time delay," Fractional Calculus and Applied Analysis, vol. 20, no. 1, pp. 52-70, 2017.

[17] W. Zhu, W. Li, P. Zhou, and C. Yang, "Consensus of fractionalorder multi-agent systems with linear models via observer-type protocol," Neurocomputing, vol. 230, pp. 60-65, 2017.

[18] L. Chen, Y. Chai, R. Wu, and J. Yang, "Stability and stabilization of a class of nonlinear fractional-order systems with caputo derivative," IEEE Transactions on Circuits and Systems II: Express Briefs, vol. 59, no. 9, pp. 602-606, 2012. 
[19] W. Zhu and D. Cheng, "Leader-following consensus of secondorder agents with multiple time-varying delays," Automatica. A Journal of IFAC, the International Federation of Automatic Control, vol. 46, no. 12, pp. 1994-1999, 2010.

[20] M. De La Sen, "About robust stability of caputo linear fractional dynamic systems with time delays through fixed point theory," Fixed Point Theory and Applications, vol. 2011, Article ID 867932, 2011. 


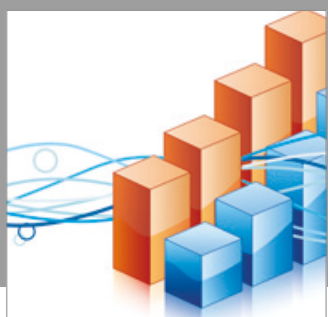

Advances in

Operations Research

vatersals

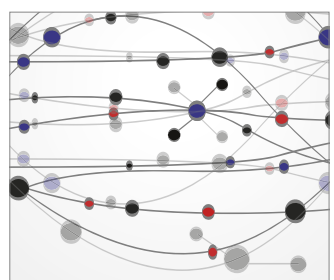

\section{The Scientific} World Journal
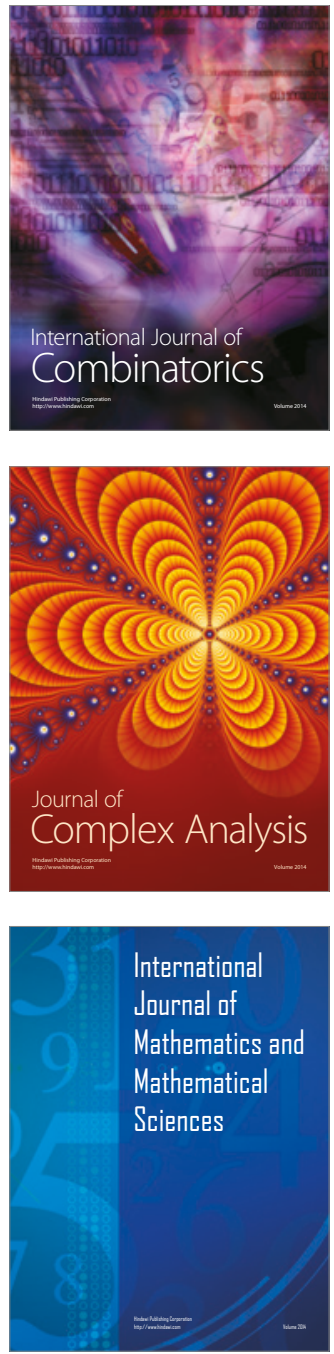
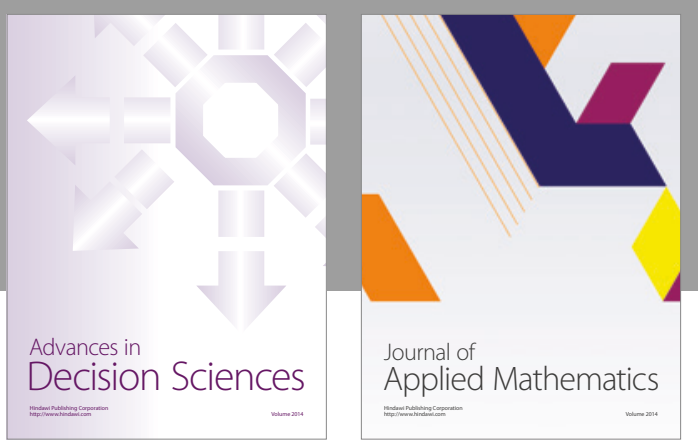

Algebra

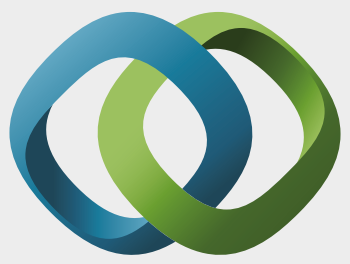

\section{Hindawi}

Submit your manuscripts at

https://www.hindawi.com
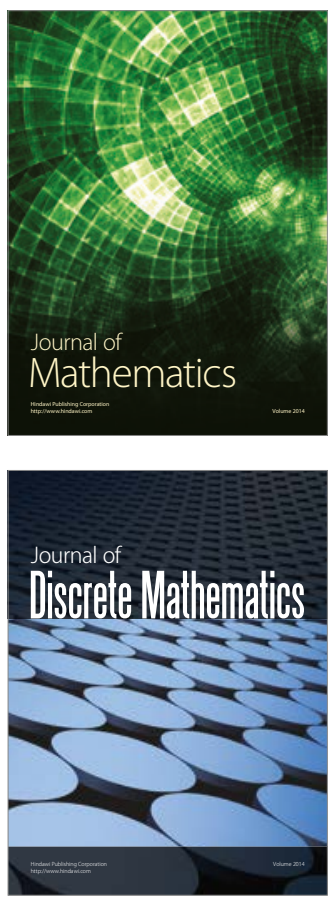

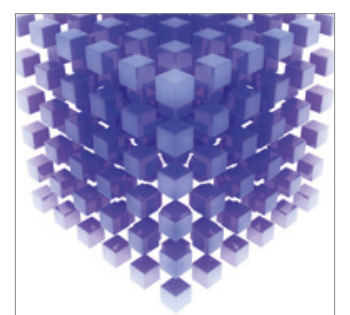

Mathematical Problems in Engineering
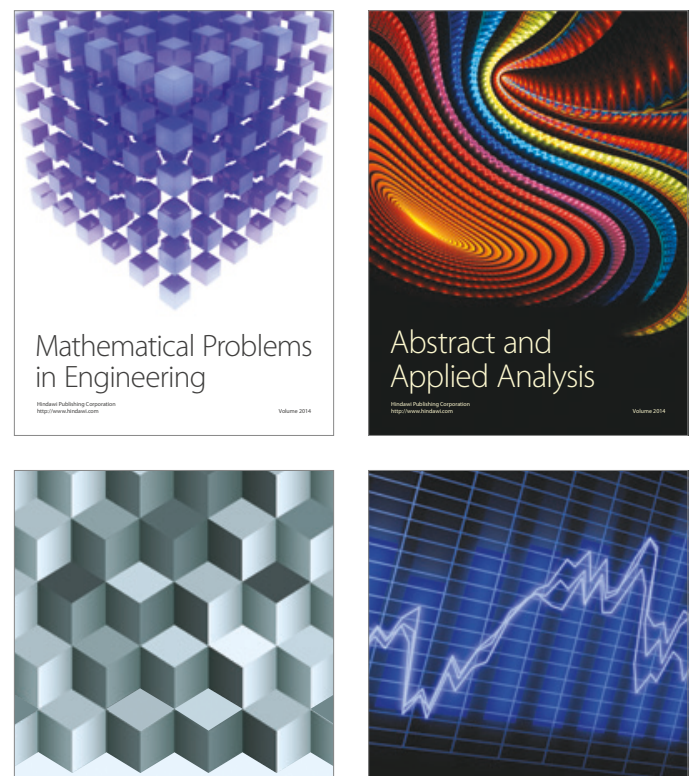

Journal of

Function Spaces

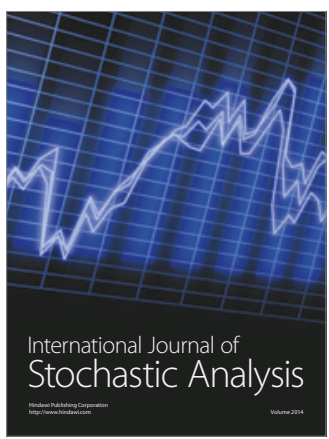

Probability and Statistics
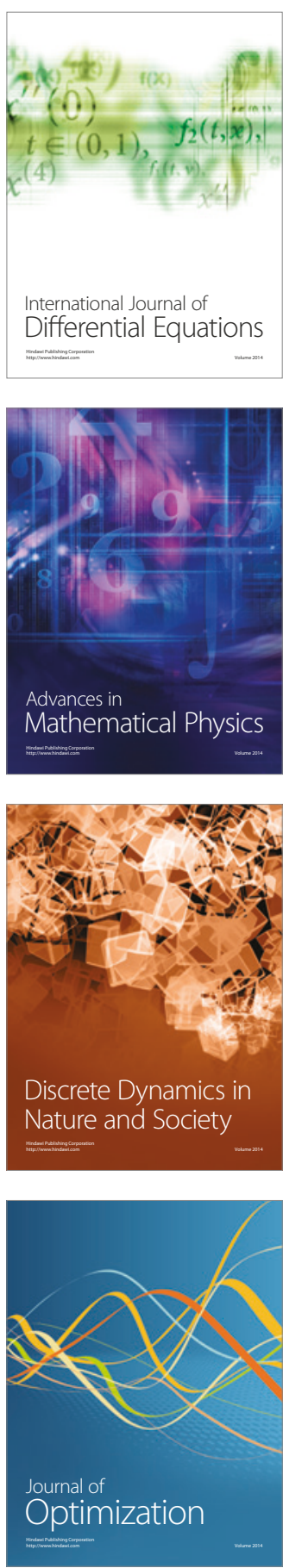